\title{
Modalidades e classificações da simulação como estratégia pedagógica em enfermagem: revisão integrativa
}

\author{
Modalities and classifications of simulation as a pedagogical strategy in nursing: \\ integrative review
}

Modalidades y clasificaciones de la simulación como estrategia pedagógica en enfermería: revisión integrativa

Isabela Meira Pereira1, Juliana da Silva Garcia Nascimento1*, Daniela da Silva Garcia Regino², Fabiana Cristina Pires ${ }^{3}$, Kleiton Gonçalves do Nascimento ${ }^{3}$, Tainá Vilhar Siqueira ${ }^{1}$, Maria Celia Barcellos Dalri'.

\section{RESUMO}

Objetivo: Compreender as modalidades e classificações da simulação como estratégia de ensino e aprendizagem para desenvolver competências clínicas em enfermagem. Métodos: Revisão integrativa, realizada em junho de 2021, nas fontes: Medical Literature Analysis and Retrieval System Online, Cumulative Index to Nursing and Allied Health Literature, Literatura Latino-Americana e do Caribe em Ciências da Saúde, Web of Science, Scopus e Educational Resources Information Center, sustentada pelo programa Rayyan para seleção dos manuscritos e pelo referencial da Análise de Conteúdo. Resultados: Nas bases de dados identificaram-se 1271 artigos e selecionaram-se oito para a amostra final. Elaboraram-se duas categorias: modalidades e classificações da simulação e habilidades desenvolvidas pela simulação. Destacaram-se a simulação clínica; virtual; híbrida e multimodal, classificadas de acordo com o ambiente de simulação, instrumentos adotados, categoria profissional envolvida e nível de fidelidade do cenário. A simulação virtual viabiliza-se por computador, jogos sérios e telessimulação. As principais habilidades desenvolvidas são cognitivas (conhecimentos), psicomotoras (procedimentais) e atitudinais (sentimentos). Considerações finais: A simulação clínica é a modalidade usualmente adotada para o ensino na enfermagem e há uma crescente valorização dos aspectos atitudinais/afetivos dos aprendizes na simulação.

Palavras-chave: Simulação, Educação em enfermagem, Competência clínica, Ensino, Aprendizagem.

\begin{abstract}
Objective: To understand the modalities and classifications of simulation as a teaching and learning strategy to develop clinical skills in nursing. Methods: Integrative review, carried out in June 2021, in the sources: Medical Literature Analysis and Retrieval System Online, Cumulative Index to Nursing and Allied Health Literature, Latin American and Caribbean Literature in Health Sciences, Web of Science, Scopus and Educational Resources Information Center, supported by the Rayyan program for manuscript selection and by the Content Analysis framework. Results: In the databases were identified1271 manuscripts and eight were selected for the final sample. Two categories were created: simulation modalities and classifications and skills developed by simulation. Clinical simulation stood out; virtual; hybrid and multimodal, classified according to the simulation environment, instruments adopted, professional category involved and level of fidelity of the
\end{abstract}

\footnotetext{
${ }^{1}$ Universidade de São Paulo (USP), Ribeirão Preto - SP. *E-mail: mestradounesp28@yahoo.com.br

2 Universidade Brasil (UB), Fernandópolis - SP.

3 Universidade Federal do Triângulo Mineiro (UFTM), Uberaba - MG.
}

SUBMETIDO EM: 9/2021

ACEITO EM: 9/2021

PUBLICADO EM: 9/2021 
scenario. Virtual simulation is made possible by computer, serious games and telesimulation. The main skills developed are cognitive (knowledge), psychomotor (procedural) and attitudinal (feelings). Final considerations: Clinical simulation is the most adopted modality for teaching in nursing and there is a growing appreciation of the assessment of attitudinal aspects of learners through simulation.

Key words: Simulation, Nursing education, Clinical competence, Teaching, Learning.

\section{RESUMEN}

Objetivo: Comprender las modalidades y clasificaciones de la simulación como estrategia de enseñanza y aprendizaje para el desarrollo de habilidades clínicas en enfermería. Métodos: Revisión integradora, realizada en junio de 2021, en fuentes: Sistema de Análisis y Recuperación de Literatura Médica en Línea, Índice Acumulado de Literatura de Enfermería y Afines en Salud, Literatura Latinoamericana y del Caribe en Ciencias de la Salud, Web of Science, Scopus e Información de Recursos Educativos Center, apoyado por programa Rayyan para selección de manuscritos y por marco de análisis de contenido. Resultados: En las bases de datos se identificaron 1271 manuscritos y seleccionaron ocho para muestra final. Se crearon dos categorías: modalidades de simulación y clasificaciones y habilidades desarrolladas por simulación. Se destacó la simulación clínica; virtual; híbrido y multimodal, clasificados según el entorno de simulación, instrumentos adoptados, categoría profesional involucrada y nivel de fidelidad del escenario. La simulación virtual es posible gracias a computadora, los juegos serios y la telesimulación. Las principales habilidades desarrolladas son cognitivas (conocimientos), psicomotoras (procedimentales) y actitudinales (sentimientos). Consideraciones finales: La simulación clínica es la modalidad más adoptada para la enseñanza en enfermería y existe una apreciación creciente de la evaluación de los aspectos actitudinales de los educandos a través de la simulación.

Palabras clave: Simulación, Educación en enfermería, Competencia clínica, Enseñando, Aprendiendo.

\section{INTRODUÇÃO}

O processo de ensino e aprendizagem em enfermagem vêm sofrendo transformações que visam atender as peculiaridades dos estudantes na contemporaneidade, mais participativos em suas atividades (MCENROE-PETITTE D e FARRIS C, 2020). Baseados nesta premissa, os docentes em enfermagem, buscam caminhos pedagógicos pautados em estratégias que valorizam o protagonismo dos alunos (FONSECA VMF e GÓMEZ J, 2017).

Destaca-se, neste contexto a simulação, caracterizada como uma estratégia de ensino e aprendizagem que imita situações reais, em ambiente seguro e controlado, dividida em três etapas, a preparação, participação e o debriefing. A preparação abrange duas fases; a pré-simulação que instrumentaliza o aprendiz por meio de estudos prévios e treinamento de habilidades e o pré-briefing/briefing, responsável pela orientação sobre o cenário simulado. Já, a etapa de participação envolve a execução do cenário clínico e o debriefing, caracteriza-se por um processo analítico de discussão/reflexão sobre a experiência, considerado fundamental para consolidar o aprendizado (TYERMAN J, et al., 2019).

Concomitante ao crescimento exponencial do uso da simulação no âmbito da enfermagem, na última década, emergiu uma variedade de nomenclaturas para caracterizar os tipos de simulação, no entanto, não foram desenvolvidos, ainda, estudos capazes de sintetizar as principais modalidades da simulação adotadas para o processo de ensino e aprendizagem em enfermagem e as suas classificações, condição que pode prejudicar a compreensão de docentes e facilitadores sobre este contexto e interferir na escolha das melhores práticas e alcance dos objetivos educacionais pretendidos (RUTHERFORD-HEMMING T, et al., 2019; TAIT L, et al., 2018; MOURA GMSS e OLSCHOWSKY A, 2017).

Diante desta lacuna científica, questionou-se: quais são as principais modalidades e classificações da simulação, adotadas para viabilizar o processo de ensino e aprendizagem em enfermagem? Este estudo 
objetivou compreender as modalidades e classificações da simulação como estratégia de ensino e aprendizagem para desenvolver competências clínicas em enfermagem.

\section{MÉTODOS}

Trata-se de uma revisão integrativa da literatura, realizada em uma universidade pública do interior do estado de São Paulo, Brasil, sustentada pela recomendação Preferred Reporting Items for Systematic Reviews and Meta-Analyses (PRISMA) (PAGE M, et al., 2020).

Percorreram-se as etapas: (1) identificação do tema e questão norteadora de pesquisa; (2) busca e seleção dos estudos; (3) categorização dos achados; (4) análise dos estudos; (5) apresentação da revisão (WHITTEMORE R E KNAFL K, 2005).

Estruturou-se a questão de pesquisa por meio da estratégia Patient-Intervention-Outcomes (PIO), uma variação da estratégia Patient-Intervention-Comparation-Outcomes (PICO) (SANTOS CM, et al., 2005). Definiu-se como acrônimo $P$ (população) estudantes e profissionais da enfermagem, como acrônimo I (intervenção) a identificação das principais modalidades e classificações da simulação em enfermagem e o acrônimo $\mathrm{O}$ (desfecho) o desenvolvimento de competências clínicas em enfermagem. Obteve-se a seguinte pergunta: Quais as evidências científicas presentes na literatura sobre as principais modalidades da simulação e suas classificações, adotadas para o desenvolvimento de competências clínicas em estudantes e profissionais de enfermagem?

A busca das evidências científicas ocorreu em junho de 2021, nas seguintes bases de dados: Medical Literature Analysis and Retrieval System Online (PubMed ${ }^{\circledR} /$ MEDLINE) Cumulative Index to Nursing and Allied Health Literature (CINAHL), Literatura Latino-Americana e do Caribe em Ciências da Saúde (LILACS), Web of Science, Scopus e Educational Resources Information Center (ERIC).

Na PubMed ${ }^{\circledR} /$ MEDLINE e Scopus foram identificados os descritores controlados no Medical Subjects Headings (MeSH), na língua inglesa: Nurses; "Students, Nursing"; "Simulation Training" e "Clinical Competence", e adotadas as palavras-chave: "simulation types"; "simulation modalities" e "simulation classification". Utilizou-se a estratégia: (Nurses OR Nurse OR "Personnel, Nursing" OR "Nursing Personnel" OR "Registered Nurses" OR "Nurse, Registered" OR "Nurses, Registered" OR "Registered Nurse" AND "Students, Nursing" OR "Pupil Nurses" OR "Student, Nursing" OR "Nurses, Pupil" OR "Nurse, Pupil" OR "Pupil Nurse" OR "Nursing Student" OR "Nursing Students" AND "Simulation Training" OR "Training, Simulation" OR "Interactive Learning" OR "Learning, Interactive" AND "Simulation Types" OR "Simulation Modalities" OR "Simulation Classification" AND "Clinical Competence" OR "Competency, Clinical" OR "Competence, Clinical" $O R$ "Clinical Competency" OR "Clinical Competencies" OR "Competencies, Clinical" OR "Clinical Skill" OR "Skill, Clinical" OR "Skills, Clinical" OR "Clinical Skills").

$\mathrm{Na}$ CINAHL identificou-se os descritores controlados em Títulos/Assuntos, na língua inglesa: Nurses; "Students, Nursing"; "Simulation Training" e "Clinical Competence". Adotaram-se as palavras-chave: "simulation types"; "simulation modalities" e "simulation classification". Realizou-se a estratégia: (Nurses AND "Students, Nursing" OR "Students, Nurse Midwifery" OR "Students, Nursing, Associate" AND Simulations AND "Simulation Types" OR "Simulation Modalities" OR "Simulation Classification" AND "Clinical Competence").

Na LILACS, os descritores controlados estavam presentes no Descritores de Ciências da Saúde (Decs), em português: Enfermeiras e Enfermeiros"; "Estudantes de Enfermagem"; "Treinamento por Simulação" e Competência Clínica". Adotaram-se as palavras-chave: "Tipos de Simulação"; "Modalidades de Simulação" e "Classificação da Simulação", e suas versões em ingles e espanhol. Realizou-se a estratégia: ("Enfermeiras e Enfermeiros" AND "Estudantes de Enfermagem" AND "Treinamento por Simulação" AND "Tipos de Simulação" OR "Modalidades de Simulação" OR "Classificação da Simulação" AND "Competência Clínica"); Em inglês: (Nurses AND "Students, Nursing" AND "Simulation Training" AND "Simulation Types" OR "Simulation Modalities" OR "Simulation Classification" AND "Clinical Competence") e em espanhol: ("Enfermeras y Enfermeros" AND "Estudiantes de Enfermería" AND "Entrenamiento Simulado" AND "Tipos de simulación" OR "Modos de simulación" OR "Clasificación de la Simulación" AND “Competencia Clínica"). 
$\mathrm{Na}$ web of science utilizaram-se os descritores controlados em inglês: Nurses; "Students, Nursing"; "Simulation Training"; "Clinical Competence" e as palavras-chave: "Simulation Types"; "Simulation Modalities" e "Simulation Classification. Adotou-se a estratégia: TS=(Nurses AND "Students, Nursing" AND "Simulation Training" AND "Simulation Types" OR "Simulation Modalities" OR "Simulation Classification" AND "Clinical Competence").

Na ERIC utilizaram-se os descritores controlados em inglês Nurses; "Students, Nursing"; "Simulation Training"; "Clinical Competence" e as palavras-chave: "Simulation Types"; "Simulation Modalities" e "Simulation Classification. Procedeu-se a estratégia: (Nurses AND "Students, Nursing" AND "Simulation Training" AND "Simulation Types" OR "Simulation Modalities" OR "Simulation Classification" AND "Clinical Competence").

Incluíram-se estudos primários, que abordavam com clareza as definições de modalidades ou classificação da simulação, sem delimitar recorte temporal e idioma, publicados em periódicos científicos, de forma eletrônica. Excluíram-se revisões da literatura, estudos de caso, dissertações, teses, monografias, resumos publicados em anais de eventos.

Foram percorridas três fases de seleção. Na primeira, realizou-se a leitura de títulos e resumos dos manuscritos, por dois profissionais, separadamente, experts na temática da simulação e subsidiados por um programa de revisão, gratuito da web, chamado Rayyan Qatar Computing Research Institute (Rayyan QCRI). O Rayyan propicia à exportação dos estudos, da fonte de informação, para o referido programa, de maneira organizada, e mantém o cegamento do pesquisador auxiliar (OUZZANI M, et al., 2016).

Na segunda fase, enviaram-se 27 estudos para um terceiro expert, por apresentarem divergência entre os primeiros pesquisadores. Este expert foi responsável por incluir ou excluir os manuscritos. Na terceira fase, avaliou-se os textos completos, para definir a amostra final. A extração das evidências ocorreu por meio de um instrumento validado considerando-se: autores; ano de publicação, país de origem do estudo; objetivos, tipo de estudo, resultados e conclusão (URSI ES e GALVÃO CM, 2006).

Ainda, classificou-se o nível de evidência dos artigos (MELNYK BM e FINEOUT-OVERHOLT E, 2011). Os achados foram analisados por meio da Análise Temática em três etapas: (1) pré-análise: leitura flutuante das evidências e organização das informações convergentes, denominadas unidades de registro; (2) agrupamento das unidades de registro; (3) determinação das categorias (MINAYO MC, 2017).

\section{RESULTADOS}

Identificaram-se, inicialmente, 1271 manuscritos e demonstrou-se sua seleção por meio do PRISMA, conforme apresentado na Figura 1. 
Figura 1 - Fluxograma de identificação, seleção e inclusão dos estudos, elaborado a partir da recomendação PRISMA.

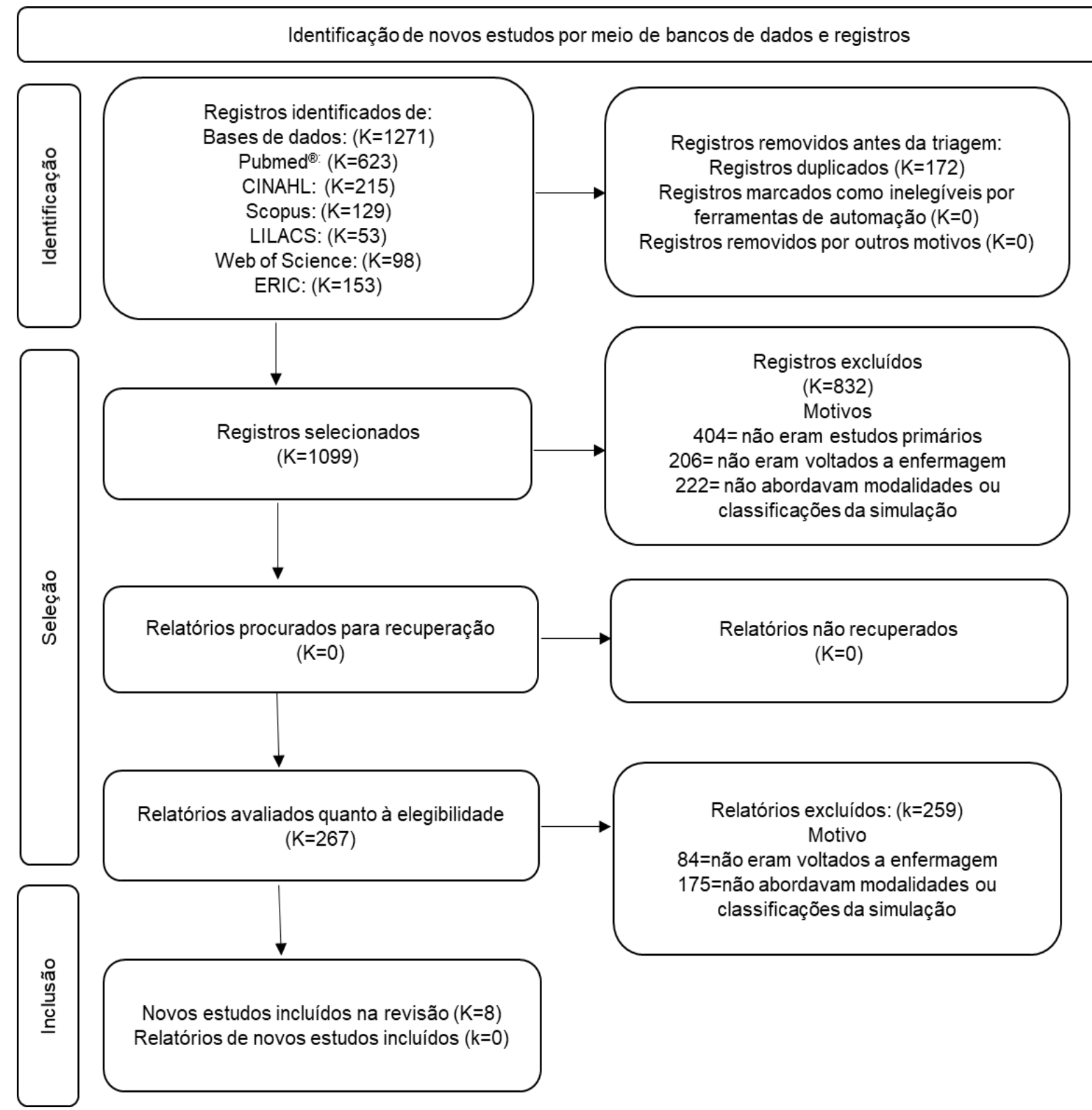

Fonte: NASCIMENTO JSG, et al., 2021.

O Quadro 1, apresenta a caracterização dos estudos incluídos na amostra da presente pesquisa, abordando os autores do estudo, ano de publicação, objetivos, tipo de estudo, principais resultados, conclusões e o nível de evidência. 
Quadro 1 - Caracterização dos estudos incluídos na amostra da presente revisão integrativa.

\begin{tabular}{|c|c|c|}
\hline Autores e ano & $\begin{array}{l}\text { Objetivo e tipo de } \\
\text { estudo }\end{array}$ & Resultados, conclusão e nível de evidência. \\
\hline $\begin{array}{l}\text { LEVE } \\
\mathrm{T}, \mathrm{e}\end{array}$ & $\begin{array}{l}\text { Comparar o conhecimento de } \\
\text { estudantes de enfermagem, } \\
\text { expostos a simulação clínica, } \\
\text { com manequins de média e } \\
\text { alta fidelidade. Estudo quase- } \\
\text { experimental }\end{array}$ & $\begin{array}{l}\text { Não houveram diferenças entre os grupos para o } \\
\text { conhecimento. Concluiu-se que, é preciso refletir } \\
\text { sobre a necessidade de se investir em simulações de } \\
\text { alta fidelidade, visto que, pode obter resultados de } \\
\text { aprendizagem semelhantes a outros níveis de } \\
\text { fidelidade. Nível de evidência 3. }\end{array}$ \\
\hline $\begin{array}{l}\text { LIAW SY, et al., } \\
2014 .\end{array}$ & $\begin{array}{l}\text { Descrever a simulação virtual } \\
\text { e compará-la com a } \\
\text { simulação clínica, quanto a } \\
\text { avaliação da deterioração } \\
\text { clínica. Estudo experimental } \\
\text { e randomizado. }\end{array}$ & $\begin{array}{l}\text { O grupo experimental (simulação virtual) e controle } \\
\text { (simulação clínica) demonstraram melhora do } \\
\text { conhecimento }(p<0,001) \text {. Concluiu-se que, ambas as } \\
\text { estratégias são eficazes para o processo de ensino } \\
\text { em enfermagem. Nível de evidência } 2\end{array}$ \\
\hline $\begin{array}{r}\text { EYIKARA } \\
\text { BAYKARA } \\
2017 .\end{array}$ & $\begin{array}{l}\text { Descrever a importância da } \\
\text { simulação. Estudo descritivo }\end{array}$ & $\begin{array}{l}\text { Estudantes de enfermagem que participam de } \\
\text { simulações promovem menos erros em ambientes } \\
\text { clínicos e melhoram o pensamento crítico. A } \\
\text { simulação foi definida de acordo com o nível de } \\
\text { fidelidade. Concluiu-se que, habilidades cognitivas, } \\
\text { psicomotoras e afetivas em enfermagem podem ser } \\
\text { potencializadas utilizando a simulação. Nível de } \\
\text { evidência } 6 \text {. }\end{array}$ \\
\hline $\begin{array}{c}\text { ZITZELSBERGER } \\
\text { H, et al., } \\
2017 .\end{array}$ & $\begin{array}{l}\text { Investigar a utilização das } \\
\text { simulações utilizadas na } \\
\text { graduação em enfermagem. } \\
\text { Estudo } \quad \text { descritivo- } \\
\text { exploratório. }\end{array}$ & $\begin{array}{l}\text { Há uma forte tendência em aumentar as experiências } \\
\text { de simulação para os alunos, a fim de prepará-los } \\
\text { para a prática em ambientes complexos e aumentar } \\
\text { a segurança do paciente. Conclui-se que, até } 50 \% \\
\text { das horas clínicas podem ser substituídas pela } \\
\text { simulação. Nível de evidência } 6 \text {. }\end{array}$ \\
\hline $\begin{array}{r}\text { UNVER } \\
20\end{array}$ & $\begin{array}{l}\text { Analisar os efeitos de uma } \\
\text { simulação híbrida com } \\
\text { estudantes de enfermagem } \\
\text { em um cenário de } \\
\text { emergência. Estudo quase- } \\
\text { experimental }\end{array}$ & $\begin{array}{l}\text { Quase todos os participantes relataram que a } \\
\text { simulação melhorou seu pensamento crítico, decisão } \\
\text { e autoconfiança, antes da atividade clínica. Concluiu- } \\
\text { se que, os resultados deste estudo apoiam a } \\
\text { integração de experiências de simulação híbrida em } \\
\text { enfermagem. Nível de evidência } 3 \text {. }\end{array}$ \\
\hline $\begin{array}{r}\text { PAPAN } \\
\text { D, } 2\end{array}$ & $\begin{array}{l}\text { Descrever a telessimulação e } \\
\text { sua aplicabilidade. Estudo } \\
\text { descritivo. }\end{array}$ & $\begin{array}{l}\text { A liga nacional de enfermagem americana identificou } \\
\text { que, um corpo docente qualificado é o componente } \\
\text { principal para a qualidade da simulação. A } \\
\text { telessimulação oferece a conveniência da } \\
\text { participação remota, alarga o acesso aos cuidados de } \\
\text { saúde; aumenta a eficiência, reduz custos; aumenta } \\
\text { a segurança do paciente e a qualidade do } \\
\text { atendimento. Os instrutores precisam estar atentos } \\
\text { ao público de aluno antes de qualquer alteração no } \\
\text { design instrucional. Nível de evidência } 6 \text {. }\end{array}$ \\
\hline $\begin{array}{l}\text { LEE C, et al., } \\
2019 .\end{array}$ & $\begin{array}{l}\text { Desenvolver um programa de } \\
\text { simulação in situ e o seu } \\
\text { impacto na confiança dos } \\
\text { enfermeiros, na deterioração. } \\
\text { Estudo quase-experimental. }\end{array}$ & $\begin{array}{l}\text { Um total de } 176 \text { enfermeiros participaram da } \\
\text { simulação in situ. Quanto a confiança do participante, } \\
\text { os mesmos se sentiram mais confiantes após a } \\
\text { simulação. Além disso, os enfermeiros descreveram } \\
\text { sentir-se seguros para reconhecer a deterioração e } \\
\text { iniciar as intervenções. Nível de evidência 3. }\end{array}$ \\
\hline $\begin{array}{l}\text { FINDIK UY, et al., } \\
2019 .\end{array}$ & $\begin{array}{l}\text { Apreender a opinião dos } \\
\text { estudantes de enfermagem } \\
\text { sobre a fidelidade do cenário. } \\
\text { Estudo descritivo e } \\
\text { exploratório }\end{array}$ & $\begin{array}{l}\text { Os alunos acreditam que, a simulação de baixa } \\
\text { fidelidade é uma estratégia capaz de aperfeiçoar as } \\
\text { suas habilidades cognitivas, psicomotoras e } \\
\text { atitudinais em enfermagem. Concluiu-se que, é } \\
\text { possível recomendar a simulação de baixa fidelidade. } \\
\text { Nível de evidência } 6 \text {. }\end{array}$ \\
\hline
\end{tabular}

Fonte: NASCIMENTO JSG, et al., 2021. 
Foram elaboradas duas categorias: (1) principais modalidades e classificações da simulação em enfermagem e (2) habilidades desenvolvidas por meio da simulação em enfermagem. Na primeira, destacaram-se as seguintes modalidades da simulação em enfermagem: (1) simulação clínica, (2) simulação virtual, (3) simulação híbrida e (4) simulação multimodal (LEVETT-JONES T, et al., 2011; LIAW SY, et al., 2014; EYIKARA E e BAYKARA ZG, 2017; ZITZELSBERGER H, et al., 2017; UNVER V, et al., 2017; PAPANAGNOU D, 2017; LEE C, et al., 2019; FINDIK UY, et al., 2019).

Todas as modalidades de simulação apresentadas podem ser classificadas de acordo com: (1) o ambiente de simulação - simulação off-site; simulação intra-hospitalar, porém fora do local de prática e simulação in situ; (2) de acordo com o instrumento que viabiliza a simulação - simulação cênica do tipo role play, do tipo paciente simulado e paciente padronizado, e simulação com manequim de baixa, média e alta fidelidade; (3) de acordo com a categoria profissional - com uma categoria profissional, interprofissional e multiprofissional; (4) de acordo com a fidelidade do cenário - baixa, média e alta fidelidade (LEVETT-JONES T, et al., 2011; LIAW SY, et al., 2014; EYIKARA E e BAYKARA ZG, 2017; ZITZELSBERGER H, et al., 2017; UNVER V, et al., 2017; PAPANAGNOU D, 2017; LEE C, et al., 2019; FINDIK UY, et al., 2019).

Ainda, a simulação virtual abrange três classificações: (1) por computador; (2) por meio de jogos sérios ou serious game e (3) telessimulação (UNVER V, et al., 2017; PAPANAGNOU D, 2017). A Figura 2, apresenta as principais modalidades da simulação adotadas para o processo de ensino e aprendizagem em enfermagem e suas respectivas classificações.

O Quadro 2, detalha as definições e as peculiaridades das modalidades da simulação e suas classificações, adotadas para o ensino em enfermagem, identificadas e sintetizadas para o presente estudo. 
Figura 2 - Apresentação das modalidades da simulação em enfermagem e suas respectivas classificações.

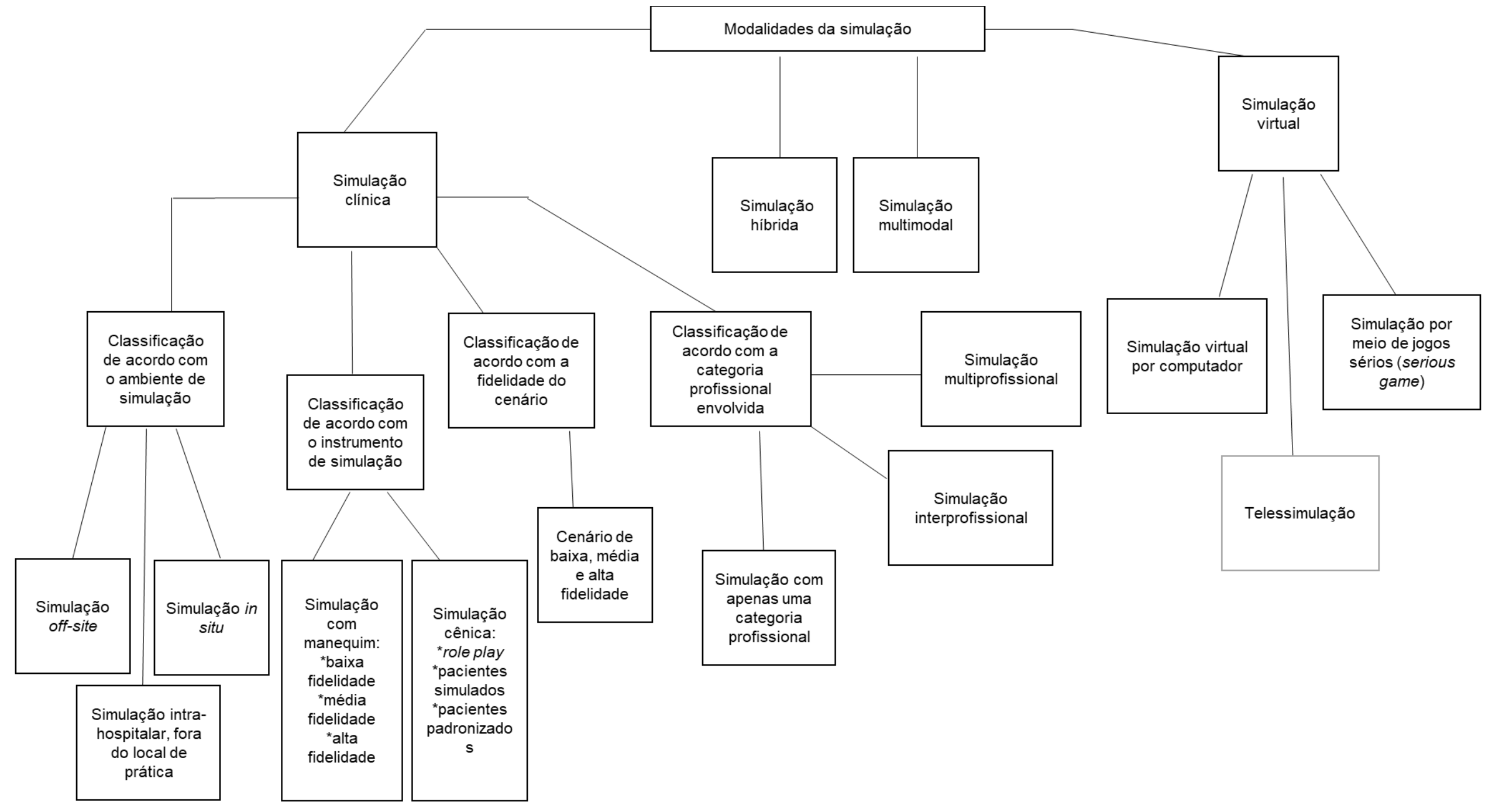

Fonte: NASCIMENTO JSG, et al., 2021. 
Quadro 2 - Apresentação da caracterização das modalidades e classificações da simulação voltada à enfermagem

\begin{tabular}{|c|c|}
\hline $\begin{array}{l}\text { Modalidades e classificações da } \\
\text { simulação }\end{array}$ & Definição \\
\hline Simulação clínica & $\begin{array}{l}\text { Estratégia de ensino e aprendizagem que imita situações clínicas reais, permitindo que, estudantes e profissionais de } \\
\text { enfermagem vivenciem situações simples ou complexas, em ambientes seguros, antes da prática (LEE C, et al., 2019). }\end{array}$ \\
\hline Simulação clínica off-site & $\begin{array}{l}\text { Simulação clínica que ocorre fora de ambientes de saúde, como por exemplo, em laboratórios de habilidades clínicas e } \\
\text { centros de simulação (LEE C, et al., 2019). }\end{array}$ \\
\hline $\begin{array}{l}\text { Simulação clínica intra-hospitalar, fora } \\
\text { do local de prática }\end{array}$ & $\begin{array}{l}\text { Simulação clínica que ocorre no ambiente de saúde, porém fora da unidade em que o cuidado é aplicado, por exemplo } \\
\text { em uma sala de aula no hospital, centro de simulação do hospital ou anfiteatro (LEE C, et al., 2019). }\end{array}$ \\
\hline Simulação clínica in situ & $\begin{array}{l}\text { Simulação clínica realizada diretamente na unidade ou setor de saúde em que a assistência de enfermagem ou cuidado } \\
\text { é prestado (LEE C, et al., 2019). }\end{array}$ \\
\hline $\begin{array}{l}\text { Simulação clínica com } \\
\text { manequim/simuladores: } \\
\text {-Baixa fidelidade } \\
\text {-Média fidelidade } \\
\text {-Alta fidelidade }\end{array}$ & $\begin{array}{l}\text {-Simuladores de baixa fidelidade: configuram-se como manequins ou peças estáticas, geralmente utilizadas para } \\
\text { procedimentos específicos, como exemplo, as peças de espuma para o treino de habilidades de injeção intramuscular; } \\
\text {-Simuladores de moderada fidelidade: capazes de gerar ausculta de sons respiratórios, cardíacos, pulsação ou } \\
\text { diagnósticos; } \\
\text {-Simuladores de alta fidelidade: manequins realísticos, capazes de obter movimentação torácica, olhos funcionais, sons } \\
\text { cardíacos, pulmonares, gastrointestinais e vocais, sangramentos e secreções e reações (LEVETT-JONES T, et al., 2011; } \\
\text { LIAW SY, et al., 2014; EYIKARA E e BAYKARA ZG, 2014; ZITZELSBERGER H, et al., 2017; FINDIK UY, et al., 2019). }\end{array}$ \\
\hline $\begin{array}{l}\text { Simulação clínica cênica: } \\
\text {-Role play } \\
\text {-Pacientes simulados } \\
\text {-Pacientes padronizados }\end{array}$ & $\begin{array}{l}\text { O role play, ou jogo de papéis, consiste na situação onde o aprendiz, assume papéis diferentes no cenário simulado, } \\
\text { para fins de ensino e treinamento; } \\
\text { Pacientes simulados são atores, treinados, que assumem um papel, retratando uma história dentro da simulação; } \\
\text { Paciente padronizado (standartized patients) pode ser definido como um membro da comunidade (criança, adolescente, } \\
\text { adulto, idoso) que concordou em assumir o papel de paciente, para uma atividade de aprendizagem (LEVETT-JONES } \\
\text { T, et al., 2011; LIAW SY, et al., 2014; EYIKARA E e BAYKARA ZG, 2014; ZITZELSBERGER H, et al., 2017; FINDIK UY, } \\
\text { et al., 2019). }\end{array}$ \\
\hline $\begin{array}{l}\text { Simulação clínica com apenas uma } \\
\text { categoria profissional }\end{array}$ & $\begin{array}{l}\text { Quando a simulação clínica envolve apenas uma categoria de profissionais, como o enfermeiro, por exemplo, os } \\
\text { objetivos de aprendizagem são exclusivamente voltados para as competências inerentes a esta profissão (LEE C, et al., } \\
\text { 2019). }\end{array}$ \\
\hline
\end{tabular}




\begin{tabular}{|c|c|}
\hline $\begin{array}{l}\text { Modalidades e classificações da } \\
\text { simulação }\end{array}$ & Definição \\
\hline Simulação clínica interprofissional & $\begin{array}{l}\text { Cenários que envolvem duas categorias profissionais, sendo um dos pilares da educação em saúde, na atualidade, e } \\
\text { auxiliando em observações específicas, principalmente quanto a relação atitudinal, de hierarquia, éticas e afetivas entre } \\
\text { as profissões expostas (LEE C, et al., 2019). }\end{array}$ \\
\hline Simulação clínica multiprofissional & $\begin{array}{l}\text { A simulação clínica multiprofissional, abrange variadas categorias profissionais, durante a execução do mesmo cenário, } \\
\text { configurando um importante recurso para lapidar o trabalho em equipe e gerar reflexão sobre as diversas situações que } \\
\text { emergem deste contexto (LEE C, et al., 2019). }\end{array}$ \\
\hline Simulação virtual por computador & $\begin{array}{l}\text { As simulações baseadas em computador são programas virtuais para treinamento e avaliação de conhecimento e } \\
\text { decisões, que oferecem flexibilidade de acesso, independente de tempo e lugar (LIAW SY, et al., 2014). }\end{array}$ \\
\hline $\begin{array}{l}\text { Simulação virtual por jogos sérios } \\
\text { (serious game) }\end{array}$ & $\begin{array}{l}\text { Os jogos sérios ou serious game computadorizados, são definidos como jogos que possuem propósitos e conteúdo } \\
\text { específicos para educar, com a exposição de um mundo virtual, em que os aprendizes participam de um cenário clínico } \\
\text { na tela do computador (EYIKARA E e BAYKARA ZG, 2017) }\end{array}$ \\
\hline Telessimulação & $\begin{array}{l}\text { É um processo pelo qual os recursos de telecomunicação e simulação são utilizados para fornecer educação, } \\
\text { treinamento e/ou avaliação, aos alunos, em um local externo. Localização externa refere-se a um local distante que } \\
\text { impediria a educação sem o uso de recursos de telecomunicações (PAPANAGNOU D, 2017). }\end{array}$ \\
\hline Simulação híbrida & $\begin{array}{l}\text { Simulação que utiliza duas modalidades diferentes, de forma combinada, para que um tipo aprimore e potencialize os } \\
\text { resultados de aprendizagem do outro (UNVER V, et al., 2017). }\end{array}$ \\
\hline Simulação multimodal & Simulação que abrange várias modalidades de uma só vez, no mesmo cenário (UNVER V, et al., 2017). \\
\hline
\end{tabular}

Fonte: NASCIMENTO JSG, et al., 2021. 
A modalidade de simulação mais explorada pela literatura em enfermagem é a simulação clínica e a classificação mais frequente caracteriza-se pelos instrumentos adotados, especificamente, quanto ao uso de simuladores de baixa, média e alta fidelidade (LEVETT-JONES T, et al., 2011; LIAW SY, et al., 2014; EYIKARA E e BAYKARA ZG, 2014; ZITZELSBERGER H, et al., 2017; FINDIK UY, et al., 2019).

A segunda categoria destacou as principais habilidades desenvolvidas por meio da simulação em enfermagem para desenvolver as competências clínicas, à saber: (1) habilidade cognitiva - conhecimento em enfermagem; (2) habilidades psicomotoras - habilidades procedimentais; (3) habilidades afetivas/atitudes: como principalmente, a confiança, satisfação, julgamento clínico e tomada de decisão (EYIKARA E e BAYKARA ZG, 2014; ZITZELSBERGER H, et al., 2017; FINDIK UY, et al., 2019). Cabe ressaltar que, a avaliação das habilidades afetivas/atitudes foi amplamente valorizada pela amostra de estudos selecionada na presente pesquisa (LEVETT-JONES T, et al., 2011; LIAW SY, et al., 2014; EYIKARA E e BAYKARA ZG, 2014; ZITZELSBERGER H, et al., 2017; FINDIK UY, et al., 2019).

\section{DISCUSSÃO}

Acredita-se que, cada modalidade da simulação seja capaz de sustentar diferentes objetivos de aprendizagem em enfermagem (EYIKARA E e BAYKARA ZG, 2017; ZITZELSBERGER H, et al., 2017; UNVER V, et al., 2017). Desta forma, este estudo confere ineditismo a ciência da enfermagem por sintetizar as principais modalidades da simulação adotadas para o processo de ensino e aprendizagem em enfermagem e classificá-las, favorecendo a compreensão de docentes e facilitadores de simulação e a escolha das melhores práticas educacionais, coerentes aos objetivos de aprendizagem pretendidos. Ainda, por demonstrar a valorização contemporânea dos aspectos atitudinais/comportamentais/afetivos dos aprendizes frente à simulação em enfermagem, uma habilidade que permanecia em detrimento das outras (cognitivas e procedimentais) na maioria dos estudos sobre simulação, até o presente momento (TYERMAN J, et al., 2019).

A simulação clínica vem obtendo destaque no ensino em enfermagem e considerada uma modalidade de simulação preferencial para muitos docentes por melhorar significativamente a iniciativa do enfermeiro para o enfrentamento da prática e configurar-se como uma modalidade inovadora, capaz de estimular a reflexão e permitir que os aprendizes pratiquem, repetidamente, suas habilidades e cometam erros em ambiente seguro (LEVETT-JONES T, et al., 2011; LIAW SY, et al., 2014; ZITZELSBERGER H, et al., 2017; UNVER V, et al., 2017; PAPANAGNOU D, 2017; LEE C, et al., 2019; FINDIK UY, et al., 2019; DONOVAN LM, et al., 2019; JUNG D, et al., 2017; EYIKARA E e BAYKARA ZG, 2017).

Os achados da presente revisão também revelaram uma preponderância na abordagem da fidelidade do manequim adotado para a simulação clínica (LEVETT-JONES T, et al., 2011; LIAW SY, et al., 2014; EYIKARA E e BAYKARA ZG, 2014; ZITZELSBERGER H, et al., 2017; FINDIK UY, et al., 2019). Neste ínterim, é importante esclarecer que, não se deve valorizar somente o nível de fidelidade do manequim utilizado na simulação clínica, mas também, a complexidade do cenário, compreendendo-a como o grau com que uma experiência simulada se aproxima da realidade, mensurado por um conjunto de dimensões: ambiente, equipamentos, ferramentas, simuladores, maquiagens, ruídos, adornos; fatores psicológicos: emoções, crenças e autoconsciência dos participantes; fatores sociais: motivação e metas dos participantes e instrutores; cultura do grupo; grau de abertura e confiança, bem como o modo de pensar dos participantes (NEGRI EC, et al., 2019; MACLEAN S, et al., 2017; KANEKO RMU e LOPES MHBM, 2019; OLIVEIRA SN, et al., 2018; LIOCE L, et al., 2020).

Outra perspectiva que merece relevância é a capacidade da simulação para desenvolver competências clínicas em enfermagem, e neste sentido, contrapondo uma tendência observada na literatura da ênfase na avaliação das habilidades cognitivas e psicomotoras dos aprendizes em uma simulação, destacou-se no presente estudo, a valorização das atitudes e sentimentos gerados pelos participantes, durante a vivência simulada (LEVETT-JONES T, et al., 2011).

Dois fatores principais podem justificar este novo achado, primeiramente o desenvolvimento recente de instrumentos capazes de sustentar a avaliação comportamental do aluno, durante a simulação, e a 
necessidade de avaliar as habilidades afetivas do aprendiz, na contemporaneidade, ao perceber-se que, só é possível abordar a temática das competências clínicas em enfermagem, de uma maneira ampla, valorizando-se os aspectos cognitivos (conhecimento), psicomotores (procedimental) e afetivos/comportamentais do aluno (LABRAGUE LJ, et al., 2019).

Corrobora com este contexto uma pesquisa do tipo revisão sistemática que verificou os efeitos da simulação clínica, como estratégia pedagógica, na confiança de estudantes em enfermagem e fornecei evidências baseadas em 35 estudos sobre a sua efetividade na redução da ansiedade e aumento da confiança dos participantes que vivenciam a simulação (LABRAGUE LJ, et al., 2019).

Também se assemelha a este resultado uma pesquisa quase-experimental que analisou a satisfação e a autoconfiança dos estudantes de enfermagem, a partir do uso da simulação sobre vacinação e constatou que, na simulação, os estudantes, além de aprenderem, tem a sua satisfação e a confiança aumentadas (COSTA RR, et al., 2020).

$\mathrm{Na}$ base do desenvolvimento de competência clínica em enfermagem está o raciocínio clínico, um componente essencial da prática competente de enfermagem que envolve a combinação da experiência e o conhecimento, bem como o pensamento crítico. A crescente complexidade do papel do enfermeiro no cuidado e gerenciamento na saúde, exige o desenvolvimento de raciocínio clínico, e neste ínterim, há evidências substanciais de que a simulação é uma via para desenvolver confiança, conhecimento e julgamento clínico (COSTA RR, et al., 2020).

Esta pesquisa apresentou como limitação o número incipiente de estudos que compuseram a sua amostra, capazes de definir as modalidades da simulação e apresentar as suas classificações no âmbito da enfermagem. Sugere-se o desenvolvimento de estudos experimentais, randomizados, capazes de comparar a efetividade das modalidades de simulação e suas classificações, aqui apresentadas, para o processo de ensino e aprendizagem em enfermagem.

\section{CONSIDERAÇÕES FINAIS}

As principais modalidades da simulação voltadas ao processo de ensino e aprendizagem em enfermagem, configuram-se pela simulação clínica, virtual, híbrida e multimodal. Considera-se a simulação clínica, a modalidade mais adotada, classificada de acordo com o ambiente em que ocorre; o instrumento que viabiliza está vivência; a fidelidade do cenário e a categoria profissional envolvida. As habilidades cognitivas, psicomotoras e afetivas, são a tríade que fundamenta o desenvolvimento de competência clínica na simulação em enfermagem, notando-se na contemporaneidade, uma crescente valorização dos aspectos atitudinais/afetivos dos aprendizes na simulação.

\section{REFERÊNCIAS}

1. COSTA RR, et al. Simulação clínica no desempenho cognitivo, satisfação e autoconfiança na aprendizagem: estudo quase-experimental. Acta Paulista de Enfermagem, 2020; 33: eAPE20180123.

2. DONOVAN LM, MULLEN LK. Expanding nursing simulation programs with a standardized patient protocol on therapeutic communication. Nursing Practice Today, 2019; 38: 126-31.

3. EYIKARA E, BAYKARA ZG. The importance of simulation in nursing education. World Journal on Educational Technology, 2017; 9(1): 02-07.

4. FINDIK UY, et al. Determining student nurses' opinions of the low-fidelity simulation method. Nursing Practice Today, 2019; 6(2): 71-6.

5. FONSECA VMF, GÓMEZ J. Applying Active Methodologies for Teaching Software Engineering in Computer Engineering. Revista Iberoamericana de Tecnologías del Aprendizaje, 2017; 12(4): 182-90.

6. JUNG D, et al. Development and evaluation of a clinical simulation for new graduate nurses: A multi-site pilot study. Nurse Education Today, 2017; 49: 84-9.

7. KANEKO RMU, LOPES MHBM. Cenário em simulação realística em saúde: o que é relevante para a sua elaboração? Revista da Escola de Enfermagem da USP, 2019; 53: e03453.

8. LABRAGUE LJ, et al. High-fidelity simulation and nursing students' anxiety and self-confidence: A systematic review. Nursing Forum, 2019; 54(3): 358-68. 
9. LEE C, et al. The Impact of Hospital-Based In Situ Simulation on Nurses' Recognition and Intervention of Patient Deterioration. Journal for Nurses in Professional Development, 2019; 35(1): 18-24.

10. LEVETT-JONES T, et al. Examining the impact of high and medium fidelity simulation experiences on nursing students' knowledge acquisition. Nurse Education in Practic, 2011; 11(6): 380-3.

11. LIAW SY, et al. Comparison of Virtual Patient Simulation With Mannequin-Based Simulation for Improving Clinical Performances in Assessing and Managing Clinical Deterioration: Randomized Controlled Trial. Journal of Medical Internet Research, 2014; 16(9): e214.

12. LIOCE L, et al. Healthcare Simulation Dictionary. Agency for Healthcare Research and Quality. 2020. Disponível em: https://www.ahrq.gov/sites/default/files/publications/files/sim-dictionary.pdf. Acessado em: 05 de junho de 2021.

13. MACLEAN S, et al. Use of simulated patients to develop communication skills in nursing education: An integrative review. Nurse Education Today, 2017; 48:90-8.

14. MCENROE-PETITTE D, FARRIS C. Using Gaming as an Active Teaching Strategy in Nursing Education. Teaching and Learning in Nursing, 2020; 15(1): 61-65.

15. MELNYK BM, FINEOUT-OVERHOLT E. Evidence-based practice in nursing \& healthcare: a guide to best practice. 2. ed. Philadelphia: Wolters Kluwer Health/Pippincott Williams \& Wilkins; 2011.

16. MINAYO MC. Amostragem e saturação em pesquisa qualitativa: consensos e controvérsias. Revista Pesquisa Qualitativa, 2017; 5(7): 1-12.

17. MOURA GMSS, OLSCHOWSKY A. Comunicação da produção científica e visibilidade do conhecimento na enfermagem. Revista Gaúcha de Enfermagem, 2017; 38(1).

18. NEGRI EC, et al. Construção e validação de cenário simulado para assistência de enfermagem a pacientes com colostomia. Texto \& Contexto Enfermagem, 2019; 28: e20180199.

19. OLIVEIRA SN, et al. Da teoria à prática, operacionalizando a simulação clínica no ensino de Enfermagem. Revista Brasileira de Enfermagem, 2018; 71(Suppl 4): 1896-903.

20. OUZZANI M, et al. Rayyan-a web and mobile app for systematic reviews. Systematic Reviews, $2016 ; 5(1): 210$.

21. PAGE M, et al. The PRISMA 2020 statement: an updated guideline for reporting systematic reviews. International Journal of Surgery, 2021; 88. Disponível em: https://www.bmj.com/content/372/bmj.n71. Acessado em: 05 de junho de 2021.

22. PAPANAGNOU D. Telesimulation: A Paradigm Shift for Simulation Education. AEM Education and Training Resources, 2017; 1(2): 137-39.

23. RUTHERFORD-HEMMING T, et al. A Systematic Review of the Use of Standardized Patients as a Simulation Modality in Nursing Education. Nursing Education Perspectives, 2019; 40(2).

24. SANTOS CM, et al. The pico strategy for the research question construction and evidence search. Revista LatinoAmericana Enfermagem, 2007; 15(3): 508-11.

25. TAIT L, et al. Simulation and Feedback in Health Education: A Mixed Methods Study Comparing Three Simulation Modalities. Pharmacy, 2018; 6(2):41.

26. TYERMAN J, et al. A systematic review of health care presimulation preparation and briefing effectiveness. Clinical Simulation in Nursing, 2019; 27: 12-25.

27. UNVER V, et al. Integrating simulation based learning into nursing education programs: Hybrid simulation. Technology and Health Care, 2017; 36(2): 263-70.

28. URSI ES, GALVÃO CM. Prevenção de lesões de pele no perioperatório: revisão integrativa da literatura. Revista Latino-Americana Enfermagem, 2006; 14(1): 124-31.

29. WHITTEMORE R, KNAFL K. The integrative review: updated methodology. Journal of Advanced Nursing, 2005; 52(5): 546-53.

30. ZITZELSBERGER H, et al. Exploring Simulation Utilization and Simulation Evaluation Practices and Approaches in Undergraduate Nursing Education. Journal of Nursing Education and Practice, $2017 ; 8(3)$. 